\title{
Depressive Symptoms and Quality of Life in Age-related Macular Degeneration Based on Korea National Health and Nutrition Examination Survey (KNHANES)
}

\author{
So Jung Ryu ${ }^{1}$, Won June Lee ${ }^{2}$, Leslie Bishop Tarver ${ }^{3}$, Yong Un Shin ${ }^{1}$, \\ Min Ho Kang, Mincheol Seong, ${ }^{1}$ Hee Yoon Cho \\ ${ }^{I}$ Department of Ophthalmology, Hanyang University Guri Hospital, Hanyang University College of Medicine, Guri, Korea \\ ${ }^{2}$ Department of Ophthalmology, Seoul National University Hospital, Seoul National University College of Medicine, Seoul, Korea \\ ${ }^{3}$ Department of Psychiatry, Massachusetts General Hospital, Harvard Medical School, Boston, MA, USA
}

Purpose: This study was conducted to investigate the depressive symptoms and quality of life (QOL) in patients with age-related macular degeneration (AMD) using data obtained from the Korea National Health and Nutritional Examination Survey V-2 (KNHANES V-2) conducted in 2011.

Methods: This was a population-based, cross-sectional study that selected 329 participants from the fifth $\mathrm{KN}$ HANES (2011) who were diagnosed with AMD by an ophthalmologist based on fundus photography. The prevalence of depressive symptoms and the health-related QOL (using EuroQol indices) in this cohort were also estimated. Factors associated with depressive symptoms, including socioeconomic status, QOL indices, and associated chronic diseases, were investigated using multivariate regression models.

Results: Depressive symptoms were observed more frequently in AMD patients than in non-AMD controls ( $p$ $=0.013$ ). Among the total 329 AMD participants, 65 (19.8\%) had depressive symptoms. There were 16 males (24.6\%) and 49 females (75.4\%). Upon multivariate analysis, significant factors found to be associated with depressive symptoms were female gender (odds ratio [OR], 2.082; 95\% confidence interval [Cl], 1.001 to 4.330 ), being in the "dependent" group for activities of daily living (OR, 4.638; 95\% Cl, 2.061 to 10.435), and having "some problems" in the "anxiety-depression" dimension of the EQ-5D (OR, 7.704; 95\% Cl, 1.890 to 31.408).

Conclusions: Female gender and being dependent on others for activities of daily living increased the association of depressive symptoms in this cohort of AMD participants. Screening for depressive symptoms in East Asian AMD patients with these characteristics should be an important component of their care.

Key Words: Age-related macular degeneration, Depression, Korea National Health and Nutritional Examination Survey, Quality of life

Received: August 16, 2016 Accepted: December 21, 2016

Corresponding Author: Hee Yoon Cho, MD. Department of Ophthalmology, Hanyang University Guri Hospital, Hanyang University College of Medicine, \#153 Gyeongchun-ro, Guri 11923, Korea. Tel: 82-31-560-2353, Fax: 82-31-564-9479, E-mail: hycho@hanyang.ac.kr
Age-related macular degeneration (AMD) has been recognized as one of the leading causes of visual loss in elderly people in Western countries [1,2]. The number of elderly people is increasing substantially in Asian countries, and the prevalence of AMD is also increasing in this part of the world $[3,4]$. 
The type of vision loss can lead to significant changes in a person's life, including a loss of independence, an inability to continue one's usual activities/hobbies, and increased social isolation due to decreased mobility and daily functioning. These factors are common for someone diagnosed with a chronic disease and are particularly typical in those with vision loss [5], which explains the expected association of depression and AMD.

Several studies have reported a relationship between depression and AMD, with a higher prevalence of depression in AMD cases than in the general population [6-9]. These studies are mostly from developed Western countries; we could not find any previous reports on the risk factors for depression in AMD patients in East Asian countries. Because many patients with AMD already have two major depression risk factors (advanced age and visual impairment), these covariates need to be carefully considered when evaluating depressive symptoms in this disease population.

Previously, we studied depression in Korean AMD patients before treatment with intravitreal ranibizumab injections [10]. The prevalence of depression in AMD was substantial and similar to previously reported rates. However, there were several methodological limitations. The study was a hospital-based pilot study with a limited sample size, and socioeconomic factors of the participants were not assessed.

Quality of life (QOL) in AMD patients was evaluated in several previous studies whose results indicated that the health-related and vision-related QOL were significantly associated with AMD [11-14]. However, these studies are also mostly from developed Western countries; no studies have been conducted in East Asia, particularly in Korea.

Therefore, to identify depressive symptoms and QOL among Korean AMD patients and to also identify associated factors that predispose AMD patients to develop depression, we used the data acquired from the fifth Korea National Health and Nutritional Examination Survey V-2 (KNHANES V-2) performed to sample households that represent the entire nation of Korea in 2011.

\section{Materials and Methods}

\section{Design and study population}

The KNHANES is a nationwide population-based sur- vey of the health and nutritional status of non-institutionalized Korean people. It began in 1998, and surveys were conducted in 1998, 2001, and 2005, and also in 2007-2009 and 2010-2012. A stratified, multi-stage, clustered probability design was used to select a representative sample of civilian, non-institutionalized Korean adults. The KNHANES consists of three parts: (1) a Health Interview Survey, (2) a Health Examination Survey, and (3) a Nutritional Survey. The Health Interview Survey was administered to all study participants, but the Health Examination Survey and Nutritional Survey were administered to only approximately one-third of the participants who were randomly selected from those who completed the Health Interview Survey. In all, 8,518 individuals participated in the 2011 KNHANES V-2. For our analyses, 2,888 participants were excluded because they did not finish the Health Interview Survey and/or the Health Examination Survey, and 3,790 were excluded because they were younger than 60 years. This left 1,840 people aged 60 years and older who finished the Health Interview Survey and the Health Examination Survey and were therefore eligible for our analyses (Fig. 1).

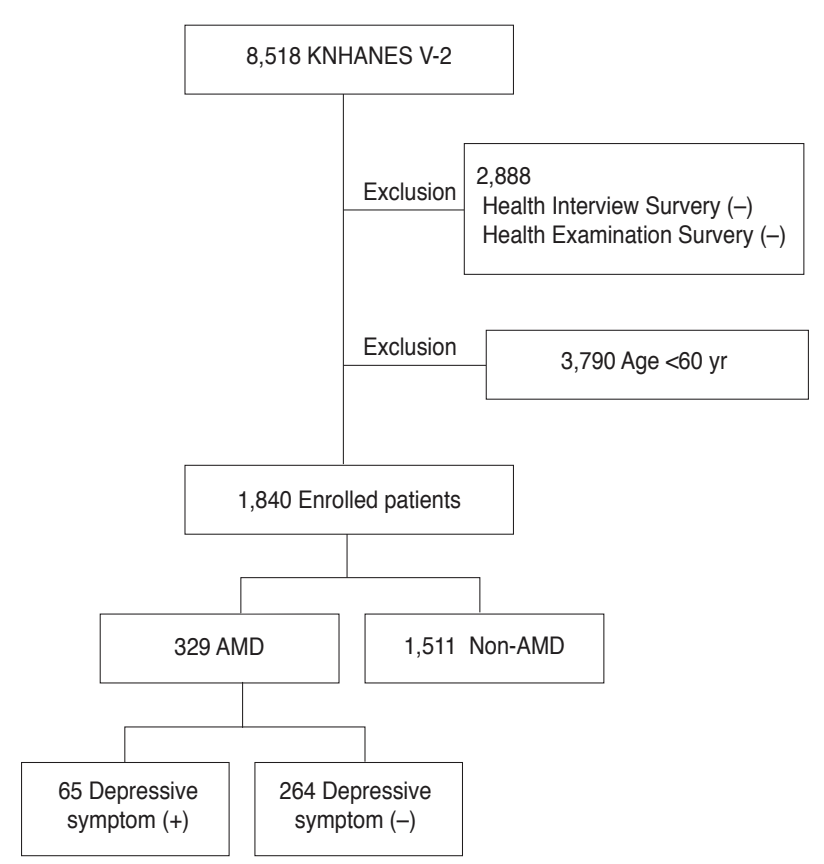

Fig. 1. Flow diagram of the study participants. KNHANES = Korea National Health and Nutrition Examination Survey; AMD $=$ age-related macular degeneration. 
This survey was reviewed and approved by the institutional review board of the Korea Centers for Disease Control and Prevention (No. 2011-02CON-06-C), and all participants provided written informed consent.

\section{Measurement}

We collected data from the KNHANES V-2 database regarding various sociodemographic factors obtained through direct interviews using standardized questionnaires.

\section{1) Depressive symptoms}

In the KNHANES V (2010-2012), the depressive symptom group was defined as those who answered "yes" to the following question: "have you felt sorrow or despair that has affected your daily life for more than 2 weeks continuously during the past year? [15]." This indicator has been previously used in other KNHANES V studies $[16,17]$.

\section{2) Demographic and health questionnaire}

The education levels were divided into "elementary school or less," "less than middle school," "high school or less," and "some university." Family income was classified into quartiles. Chronic diseases included hypertension, stroke, ischemic heart disease, osteoarthritis, and diabetes. A participant was classified as having cancer if he or she had received at least one cancer diagnosis. Each disease was defined as "yes" if the participants answered positively to "did a doctor diagnose the disease?" so as to reduce errors from subjective self-diagnoses. Smoking status was classified as "current," "ex-smoker," and "never-smoker." Drinking frequency was classified into the following groups: "less than once per month," "1-4 times per month," and "more than twice per week."

For activities of daily living (ADL), "independent" was defined as a response of "no" to the question "do you have any disturbances in everyday life?" "dependent" was defined as an answer of "yes" to this same question. The respondents' perceived health was determined using the question "how is your health in general? is it very good, good, average, bad, or very bad?" The participants were dichotomized into two groups based on their answers: one group for those who responded "very good, good, or average" and another group for those who responded "bad or very bad." The EuroQol was used to evaluate the health-re- lated QOL. The EuroQol consists of two parts: the health-status descriptive system (EQ-5D) and a visual analogue scale (EQ-VAS). The EQ-5D records the level of self-reported problems across five dimensions (mobility, self-care, usual activities, pain/discomfort, and anxiety/depression) $[18,19]$. Each of these dimensions is assessed using a single question with three response levels (no problems, some problems, and extreme problems). A single health index score was calculated from a combination of these items using the Korea valuation set developed by the Korea Centers for Disease Control and Prevention [20]. Scores on the EQ-5D index range from -0.171 to 1 , where 1 indicates no problems in any of the five dimensions, zero indicates death, and negative values suggest a health status worse than death. Finally, the respondents described their own health status using a VAS ranging from 0 (worst imaginable health) to 100 (best imaginable health) [18].

\section{3) Ophthalmic examinations and definition of AMD}

A digital non-mydriatic fundus camera (TRC-NW6S; Topcon, Tokyo, Japan) was used to obtain fundus photographs. For each participant, one $45^{\circ}$ non-mydriatic digital retinal image centered on the fovea was taken per eye for a total of two images per person. The digital images were captured under physiological mydriasis. These images were graded twice (a preliminary grade and a detailed grade) using the grading protocol of the International Age-related Maculopathy Epidemiological Study Group [21]. Patients were defined as having early AMD if they met any of the following criteria: (1) the presence of soft indistinct drusen or reticular drusen or (2) the presence of hard or soft distinct drusen with pigmentary abnormalities (increased pigmentation or hypopigmentation of the retinal pigment epithelium) in the absence of signs of late AMD. Late AMD included the presence of signs of wet AMD or geographic atrophy. Wet AMD was defined as retinal pigment epithelial detachment or serous detachment of the sensory retina, subretinal or sub-RPE hemorrhages, and subretinal fibrous scars. Geographic atrophy was defined as a circular discrete area (at least $175 \mu \mathrm{m}$ in diameter) of retinal depigmentation with visible choroidal vessels in the absence of signs of wet AMD.

The best-corrected visual acuity was measured at a distance of $4 \mathrm{~m}$ using an international standard vision chart based on the logarithm of the minimum angle of resolution (logMAR) scale (Jin's Vision Chart, Seoul, Korea) [22]. Vi- 
sual impairment was defined as a best-corrected visual acuity of 0.32 or worse in the best eye.

\section{Statistical analysis}

Descriptive statistical methods were used to describe the basic characteristics of the study population. Numbers and percentages were reported for each variable. The prevalence of depressive symptoms and the EuroQol indices in AMD patients versus non-AMD age-matched controls were estimated. Differences between those included in the analyses were assessed using the independent-samples $t$-test for continuous variables, such as age or visual acuity. For qualitative or categorical variables, such as educational status or gender, we used the chi-square test for analysis.

A two-step, multidimensional approach was used to identify the factors associated with depressive symptoms in AMD patients. First, to identify factors associated with depressive symptoms, odds ratios and $95 \%$ confidence intervals (CI) were calculated using univariate logistic regression analysis. Second, multivariate logistic regression was used to determine independent associated factors. All associated factors that were identified as affecting depressive symptoms by univariate analysis were included in the multivariate analysis to determine which factors were independently associated with depressive symptoms. All statistical tests were performed using PASW ver. 18.0 (SPSS Inc., Chicago, IL, USA).

\section{Results}

\section{The prevalence of depressive symptoms in Korean AMD patients}

Table 1 provides the baseline characteristics of the study

Table 1. Baseline characteristics of the study population and prevalence of depressive symptoms

\begin{tabular}{|c|c|c|c|}
\hline & Non-AMD control $(n=1,511)$ & $\operatorname{AMD}(\mathrm{n}=329)$ & $p$-value \\
\hline Sex & & & 0.060 \\
\hline Male & $669(44.3)$ & $127(38.6)$ & \\
\hline Female & $842(55.7)$ & $202(61.4)$ & \\
\hline Age (yr) & $69.18 \pm 6.447$ & $69.41 \pm 7.740$ & 0.575 \\
\hline Depressive symptom & & & 0.013 \\
\hline No & $1,288(85.6)$ & $264(80.2)$ & \\
\hline Yes & $216(14.4)$ & $65(19.8)$ & \\
\hline $\mathrm{ADL}$ & & & 0.060 \\
\hline Dependent & $310(20.5)$ & $83(25.2)$ & \\
\hline Independent & $1,200(79.5)$ & $246(74.8)$ & \\
\hline Perceived health & & & 0.363 \\
\hline Good & $1,067(70.6)$ & $224(68.1)$ & \\
\hline Not good & $444(29.4)$ & $105(31.9)$ & \\
\hline $\operatorname{logMAR}$ of the better eye & $0.0979 \pm 0.1400$ & $0.1139 \pm 0.1746$ & 0.123 \\
\hline $\log$ MAR of the worse eye & $0.1896 \pm 0.3358$ & $0.2290 \pm 0.4065$ & 0.066 \\
\hline Visual impairment & & & 0.012 \\
\hline No & $1,481(98.8)$ & 314 (96.9) & \\
\hline Yes & $18(1.2)$ & $10(3.1)$ & \\
\hline \multicolumn{4}{|l|}{ Quality of life } \\
\hline EQ-5D index & $0.874 \pm 0.175$ & $0.853 \pm 0.181$ & 0.060 \\
\hline EQ-VAS & $69.28 \pm 21.11$ & $67.34 \pm 22.50$ & 0.155 \\
\hline
\end{tabular}

Values are presented as number (\%) or mean \pm standard deviation.

$\mathrm{AMD}=$ age-related macular degeneration; $\mathrm{ADL}=$ activities of daily living; logMAR = logarithm of the minimum angle of resolution. 
participants and a comparison of the characteristics between AMD patients and non-AMD age-matched controls. Of the 329 AMD patients, 65 (19.8\%) exhibited depressive symptoms. Of the 1,511 non-AMD age-matched controls, $216(14.4 \%)$ had depressive symptoms $(p=0.013)$.

\section{Health-related QOL in Korean AMD patients}

The mean EQ-5D index score was 0.853 for those with AMD and 0.874 for those without AMD $(p=0.060)$. The mean EQ-VAS score was 67.34 for those with AMD and 69.28 for those without AMD $(p=0.155)$. Both indices representing the health-related QOL were not significantly different between the two groups.

The percentages of participants who had problems with each scale of the EQ-5D index are shown in Fig. 2. Patients with AMD were more likely to have difficulty with usual activities and to experience pain/discomfort than were controls without AMD. All other scales showed no significant difference between the two groups.

\section{A comparison of characteristics according to depres- sive symptoms among AMD patients}

Table 2 provides a comparison of the characteristics between AMD patients both with and without depressive symptoms. Patients with depressive symptoms were more likely to be female than patients without depressive symptoms $(75.4 \%$ vs. $58.0 \%, p=0.010)$. Patients who reported depressive symptoms were more likely to be dependent on others for their ADL than patients without depressive symptoms (56.9\% vs. $17.4 \%, p<0.001)$. Those with depressive symptoms were also more likely to perceive their health as "bad" than patients without depressive symptoms (55.4\% vs. 26.1\%, $p<0.001$ ). All EuroQol indices, including the EQ-5D and EQ-VAS, showed significant differenc-
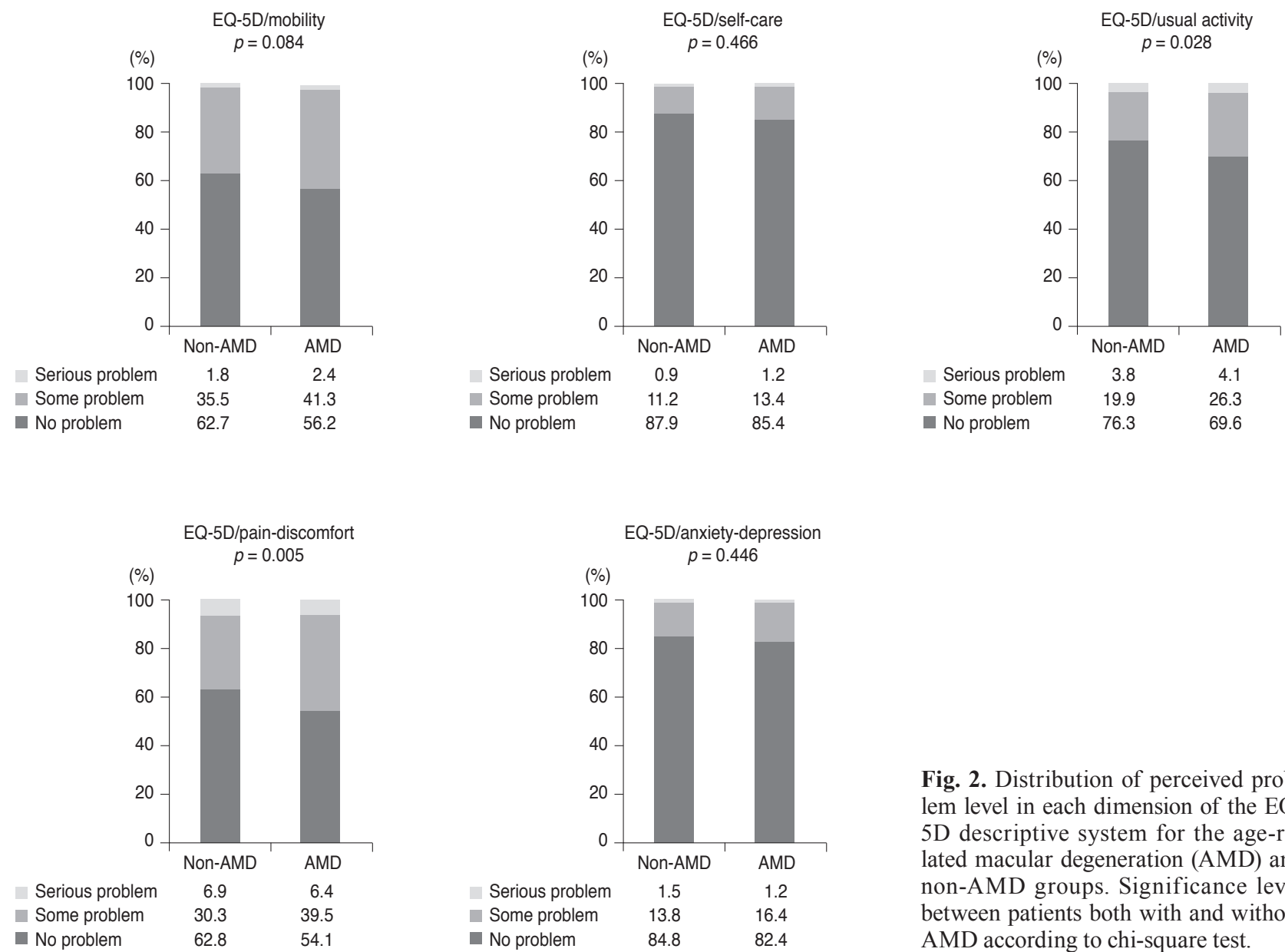

Fig. 2. Distribution of perceived problem level in each dimension of the EQ$5 \mathrm{D}$ descriptive system for the age-related macular degeneration (AMD) and non-AMD groups. Significance level between patients both with and without AMD according to chi-square test. 
Table 2. A comparison of the characteristics of AMD patients both with and without depressive symptoms

\begin{tabular}{|c|c|c|c|}
\hline & & & 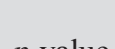 \\
\hline & No $(n=264)$ & Yes $(n=65)$ & pratue \\
\hline Sex & & & 0.010 \\
\hline Male & $111(42.0)$ & $16(24.6)$ & \\
\hline Female & $153(58.0)$ & $49(75.4)$ & \\
\hline Age (yr) & $69.8 \pm 7.712$ & $67.86 \pm 7.719$ & 0.071 \\
\hline Education & & & 0.179 \\
\hline$\leq$ Elementary school & $161(61.0)$ & $45(69.2)$ & \\
\hline$\leq$ Middle school & $33(12.5)$ & $10(15.4)$ & \\
\hline$\leq$ High school & $48(18.2)$ & $9(13.8)$ & \\
\hline$\geq$ University & $22(8.3)$ & $1(1.5)$ & \\
\hline Income & & & 0.293 \\
\hline 1st (lowest) & $77(29.7)$ & $27(42.2)$ & \\
\hline 2nd & $60(23.2)$ & $13(20.3)$ & \\
\hline $3 \mathrm{rd}$ & $68(26.3)$ & $11(20.3)$ & \\
\hline 4th (highest) & $54(20.8)$ & $11(17.2)$ & \\
\hline Hypertension & & & 0.778 \\
\hline No & $137(51.9)$ & $35(53.8)$ & \\
\hline Yes & $127(48.1)$ & $30(46.2)$ & \\
\hline Stroke & & & 0.686 \\
\hline No & $253(95.8)$ & $63(96.9)$ & \\
\hline Yes & $11(4.2)$ & $2(3.1)$ & \\
\hline IHD & & & 0.182 \\
\hline No & 248 (93.9) & $58(89.2)$ & \\
\hline Yes & $16(6.1)$ & $7(10.8)$ & \\
\hline Osteoarthritis & & & 0.076 \\
\hline No & $192(72.7)$ & $40(61.5)$ & \\
\hline Yes & $72(27.3)$ & $25(38.5)$ & \\
\hline Diabetes & & & 0.518 \\
\hline No & $235(89.0)$ & $56(86.2)$ & \\
\hline Yes & $29(11.0)$ & $9(13.8)$ & \\
\hline Cancer & & & 0.784 \\
\hline No & $254(96.2)$ & $63(96.9)$ & \\
\hline Yes & $10(3.8)$ & $2(3.1)$ & \\
\hline Smoking status & & & 0.064 \\
\hline Current smoker & $41(15.4)$ & $7(10.8)$ & \\
\hline Ex-smoker & $60(22.8)$ & $8(12.3)$ & \\
\hline Non-smoker & $162(61.6)$ & $50(76.9)$ & \\
\hline Drinking status & & & 0.890 \\
\hline$<1$ Times per month & $227(86.3)$ & $55(84.6)$ & \\
\hline 1-4 Times per month & $27(10.3)$ & $7(10.8)$ & \\
\hline$\geq 2$ Times per week & $9(3.4)$ & $3(4.6)$ & \\
\hline
\end{tabular}


Table 2. Continued

\begin{tabular}{|c|c|c|c|}
\hline & \multicolumn{2}{|c|}{ Depressive symptom } & \multirow{2}{*}{$p$-value } \\
\hline & No $(n=264)$ & Yes $(n=65)$ & \\
\hline $\mathrm{ADL}$ & & & $<0.001$ \\
\hline Dependent & $46(17.4)$ & $37(56.9)$ & \\
\hline Independent & $218(82.6)$ & $28(43.1)$ & \\
\hline Perceived health & & & $<0.001$ \\
\hline Good & 195 (73.9) & $29(44.6)$ & \\
\hline $\mathrm{Bad}$ & $69(26.1)$ & $36(55.4)$ & \\
\hline AMD grading & & & 0.944 \\
\hline Early AMD & $243(92.0)$ & $60(92.3)$ & \\
\hline Late AMD & $21(8.0)$ & $5(7.7)$ & \\
\hline $\operatorname{logMAR}$ of the better eye & $0.1207 \pm 0.1900$ & $0.0857 \pm 0.0800$ & 0.154 \\
\hline $\log$ MAR of the worse eye & $0.2418 \pm 0.4251$ & $0.1762 \pm 0.3155$ & 0.251 \\
\hline Visual impairment & & & 0.693 \\
\hline No & $252(96.6)$ & $62(98.4)$ & \\
\hline Yes & $9(3.4)$ & $1(1.6)$ & \\
\hline \multicolumn{4}{|l|}{ Quality of life } \\
\hline EQ-5D index & $0.875 \pm 0.166$ & $0.767 \pm 0.209$ & $<0.001$ \\
\hline EQ-VAS & $69.45 \pm 22.18$ & $59.22 \pm 22.11$ & 0.001 \\
\hline \multicolumn{4}{|c|}{ EQ-5D (no / some / severe problem) } \\
\hline EQ-5D/mobility & $61.2 / 36.5 / 2.3$ & $36.9 / 60.0 / 3.1$ & 0.001 \\
\hline EQ-5D/self-care & $88.2 / 11.0 / 0.8$ & $75.4 / 21.5 / 3.1$ & 0.018 \\
\hline EQ-5D/usual activity & $76.0 / 20.9 / 3.0$ & $44.6 / 47.7 / 7.7$ & $<0.001$ \\
\hline EQ-5D/pain-discomfort & $58.9 / 35.7 / 5.3$ & $35.4 / 53.8 / 10.8$ & 0.002 \\
\hline EQ-5D/anxiety-depression & $89.7 / 9.9 / 0.4$ & $53.8 / 41.5 / 4.6$ & $<0.001$ \\
\hline
\end{tabular}

Values are presented as number (\%) or mean \pm standard deviation.

$\mathrm{AMD}=$ age-related macular degeneration; $\mathrm{IHD}=$ ischemic heart disease; $\mathrm{ADL}=$ activities of daily living; logMAR $=$ logarithm of the minimum angle of resolution.

es between patients both with and without depressive symptoms. Other characteristics showed no significant differences between patients with and without depressive symptoms.

\section{Factors associated with depressive symptoms in Kore- an AMD patients}

Table 3 shows the factors associated with depressive symptoms in Korean AMD patients. Univariate logistic regression analysis determined that gender, dependency in ADL, perceived health status, and some EuroQol indices were significantly associated with depressive symptoms in Korean AMD patients. Multivariate logistic regression analysis (reported as adjusted odds ratio [aORs]) identified that gender, dependency in ADL, and having "some problems" on the "anxiety/depression" dimension of the EQ-5D were significantly and independently associated with depressive symptoms in Korean AMD patients. The results indicated a relationship between gender and depressive symptoms in Korean AMD patients: women were more likely to have depressive symptoms than men (aOR, 1.96; 95\% CI, 1.017 to 3.787). Participants who were in the ADL "dependent" group were more likely to report depressive symptoms than participants who were in the ADL "independent" group (aOR, 4.792; 95\% CI, 2.493 to 9.211). Participants with "some problems" on the "anxiety/depression" dimension of the EQ-5D were more likely to have depressive symptoms than participants reporting "no problems" on the "anxiety/depression" dimension of the EQ- 
Table 3. The univariate and multivariate analyses of factors associated with depressive symptoms in Korean AMD patients

\begin{tabular}{|c|c|c|c|c|c|}
\hline & & \multicolumn{2}{|c|}{ Univariate analysis } & \multicolumn{2}{|c|}{ Multivariate analysis } \\
\hline & & OR & $95 \% \mathrm{CI}$ & $\mathrm{aOR}^{*}$ & $95 \% \mathrm{CI}$ \\
\hline Sex $($ ref $=$ male $)$ & Female & 2.222 & $1.201-4.110^{\dagger}$ & 2.082 & $1.001-4.330^{\dagger}$ \\
\hline Age & & 0.967 & $0.933-1.003$ & - & - \\
\hline \multirow[t]{3}{*}{ Education (ref = $\geq$ university) } & $\leq$ Elementary school & 6.149 & $0.807-46.870$ & - & - \\
\hline & $\leq$ Middle school & 6.667 & $0.796-55.832$ & - & - \\
\hline & $\leq$ High school & 4.125 & $0.492-34.598$ & - & - \\
\hline \multirow[t]{3}{*}{ Income (ref = 4th [highest $])$} & 1st (lowest) & 1.721 & $0.787-3.765$ & - & - \\
\hline & 2nd & 1.064 & $0.440-2.572$ & - & - \\
\hline & $3 \mathrm{rd}$ & 0.939 & $0.390-2.260$ & - & - \\
\hline Hypertension $(\mathrm{ref}=\mathrm{no})$ & Yes & 0.925 & $0.537-1.593$ & - & - \\
\hline Stroke $($ ref $=$ no $)$ & Yes & 0.730 & $0.158-3.378$ & - & - \\
\hline IHD $($ ref = no) & Yes & 1.871 & $0.736-4.756$ & - & - \\
\hline Osteoarthritis $(\mathrm{ref}=$ no $)$ & Yes & 1.667 & $0.944-2.942$ & - & - \\
\hline Diabetes $($ ref $=$ no $)$ & Yes & 1.302 & $0.584-2.906$ & - & - \\
\hline Cancer $(\mathrm{ref}=\mathrm{no})$ & Yes & 0.086 & $0.172-3.773$ & - & - \\
\hline \multirow[t]{2}{*}{ Smoking status (ref = never-smoker) } & Current smoker & 0.553 & $0.234-1.310$ & - & - \\
\hline & Ex-smoker & 0.432 & $0.194-0.964$ & - & - \\
\hline \multirow[t]{2}{*}{ Drinking status (ref $=<1$ times per month) } & 1-4 Times per month & 1.070 & $0.443-2.585$ & - & - \\
\hline & $\geq 2$ Times per week & 1.376 & $0.360-5.251$ & - & - \\
\hline ADL (ref = independent) & Dependent & 6.262 & $3.489-11.240^{\dagger}$ & 4.638 & $2.061-10.435^{\dagger}$ \\
\hline Perceived health (ref = good) & $\mathrm{Bad}$ & 3.508 & $2.002-6.147^{\dagger}$ & 1.448 & $0.658-3.189$ \\
\hline AMD grading (ref = early) & Late AMD & 0.964 & $0.349-2.662$ & - & - \\
\hline $\log$ MAR of the better eye & & 0.093 & $0.004-1.982$ & - & - \\
\hline $\log$ MAR of the worse eye & & 0.593 & $0.238-1.479$ & - & - \\
\hline Visual impairment $(\mathrm{ref}=\mathrm{no})$ & Yes & 0.452 & $0.056-3.632$ & - & - \\
\hline \multicolumn{6}{|l|}{ Quality of life } \\
\hline EQ-5D index & & 0.057 & $0.014-0.230$ & NS & NS \\
\hline EQ-VAS index & & 0.981 & $0.969-0.992^{\dagger}$ & 0.996 & $0.971-1.006$ \\
\hline \multirow[t]{2}{*}{ EQ-5D/mobility (ref = no problems) } & Some problems & 2.725 & $1.545-4.809^{\dagger}$ & 1.712 & $0.086-22.135$ \\
\hline & Serious problems & 2.236 & $0.427-11.723$ & & \\
\hline \multirow[t]{2}{*}{ EQ-5D/self-care (ref = no problems) } & Some problems & 2.286 & $1.126-4.642^{\dagger}$ & 0.867 & $0.194-3.462$ \\
\hline & Serious problems & 4.735 & $0.651-34.431$ & & \\
\hline \multirow[t]{2}{*}{ EQ-5D/usual activity (ref = no problems) } & Some problems & 3.887 & $2.160-6.997^{\dagger}$ & 2.680 & $0.538-13.573$ \\
\hline & Serious problems & 4.310 & $1.320-14.074^{\dagger}$ & 7.019 & $0.039-1,438.920$ \\
\hline \multirow[t]{2}{*}{ EQ-5D/pain-discomfort (ref = no problems) } & Some problems & 2.509 & $1.398-4.505^{\dagger}$ & 1.102 & $0.240-5.073$ \\
\hline & Serious problems & 3.370 & $1.230-9.228^{\dagger}$ & 1.724 & $0.017-155.788$ \\
\hline \multirow[t]{2}{*}{ EQ-5D/anxiety-depression (ref = no problems) } & Some problems & 7.002 & $3.674-13.346^{\dagger}$ & 7.701 & $1.889-31.410^{\dagger}$ \\
\hline & Serious problems & 20.22 & $2.047-199.925^{\dagger}$ & 44.690 & $0.397-5,064.518$ \\
\hline
\end{tabular}

$\mathrm{AMD}=$ age-related macular degeneration; $\mathrm{OR}=$ odds ratio; $\mathrm{CI}=$ confidence interval; $\mathrm{aOR}=$ adjusted odds ratio; $\mathrm{IHD}=$ ischemic heart disease; ADL = activities of daily living; logMAR = logarithm of the minimum angle of resolution; NS = not significant.

${ }^{*}$ All associated factors that were identified as affecting depressive symptoms by univariate analysis were included in the multivariate analysis; ${ }^{\dagger} p$-value $<0.05$. 
5D. Other factors showed no significant association with depressive symptoms in Korean AMD patients.

\section{Discussion}

Using data acquired from the 2011 KNHANES V-2 nationally representative survey, we determined that the prevalence of depressive symptoms in Korean AMD patients was $19.8 \%$. We observed that the incidence of depressive symptoms in AMD patients was higher than that of non-AMD aging controls (19.8\% vs. $14.4 \%, p=0.013)$. This finding is consistent with several previous epidemiologic studies that have suggested that the prevalence of depression is higher in patients with AMD than in non-institutionalized older persons, whose rate of depression ranges between $8 \%$ and $16 \%$ [23]. The prevalence of depression in the geriatric population in Korea is generally within the range of $4.2 \%$ to $13.3 \%$ [24,25]. However, the incidence of depression in the Korean population in the present study was slightly lower than that identified by previous studies in other populations $(32.5 \%-33.0 \%$ in the United States [7,9], $21.3 \%$ in Canada [8], and $26.4 \%$ in India [6]). This finding may be partially explained by differences in the study designs and the population samples. Comparing the prevalence of depressive symptoms between different studies is often difficult because the study populations, depressive symptom measurement techniques, study methodologies, and definitions of depression are different. The present study was open to all patients with AMD in the community, and early AMD was included; other reports have limited their sample to patients recently diagnosed in outpatient retina clinics [8], those with a reduced baseline visual acuity [9], or patients who have suffered acute vision loss [7].

We also identified female gender, being in the ADL "dependent" group, and having "some problems" on the "anxiety/depression" dimension of the EQ-5D as independent associated factors for depressive symptoms in AMD patients.

Generally, the interrelationship between gender and depressive symptoms is well known. The prevalence of depression in Korea is significantly higher in women than in men [26]. However, previous studies of depression in AMD patients from Western populations have reported that gender is not significantly associated with depression in AMD patients [7-9]. Racial/ethnic differences between East Asian and Western populations may explain these disparate results.

In the present study, dependency in ADL was associated with a four-fold increase in the odds of depressive symptoms in AMD. This finding may be partially explained by the fact that a loss of vision can significantly disrupt daily life or social activity. The results of the EuroQol support this hypothesis, which showed that AMD patients were more likely to have problems with usual activities and to experience pain/discomfort than non-AMD controls. These results suggest that simply asking AMD patients about difficulty with ADL during ophthalmologic evaluations can be a valuable screening technique to identify patients at risk of depressive symptoms.

Our previous study indicated that increasing age was the most important factor associated with depression in patients with late AMD treated with intravitreal ranibizumab [10]. However, in the present population-based study, increasing age was not significantly associated with depressive symptoms in Korean AMD patients. Therefore, this finding was inconsistent with previous results. This discrepancy may be explained by differences in the study designs. The present study included early AMD as well as late AMD, whereas the previous study limited the sample to late AMD patients who had been treated with intravitreal ranibizumab. According to a previous epidemiologic study in Korea, the mean age of early AMD patients also tends to be younger than that of late AMD patients, which might have also affected the results [4].

In the current study, we expected that ophthalmic factors, including visual acuity and AMD grade, would show a strong association with depressive symptoms in AMD patients. However, the results revealed non-significant relationships between these ophthalmic factors and depressive symptoms. Previous studies have reported conflicting results with regard to visual acuity. In the literature, two studies found weak or non-significant correlations between visual acuity and depression [7,9], while two other reports concluded that visual acuity is an important determinant of depression $[6,8]$. This discrepancy may be due to differences in the sample selection process. Grade of AMD (early or late) was also not significantly associated with depressive symptoms in the present study. According to previous studies that compared Asian and Western populations, the prevalence of late AMD in Asians was compara- 
ble with that reported for Western patients, but early AMD was less common among Asians [3,4]. This racial/ethnic difference needs to be considered when interpreting these results.

To determine if the associations between female gender and dependency in ADL with depressive symptoms were specific to patients with AMD, we additionally performed a multivariate logistic regression analysis of non-AMD patients and found similar associations. Female gender and dependency in ADL were also associated with depressive symptoms in non-AMD patients (aOR, 1.785; 95\% CI, 1.298 to 2.456 ; aOR, $2.250 ; 95 \% \mathrm{CI}, 1.619$ to 3.126 , respectively).

Although these factors were not specific only in AMD patients, the results of our study can be valuable. When ophthalmologists encounter AMD patients with these associated factors, there should be more careful consideration of any depressive symptoms that may be present. Additionally, it is important to recognize that all AMD patients, regardless of the stage and degree of visual acuity, are at risk for difficulty with ADL and therefore an increased incidence of depressive symptoms. Therefore, all AMD patients should be screened for both ADL and depressive symptoms.

In the current study, health-related QOL showed no difference between AMD patients and non-AMD controls. Although general health-related QOL assessments provide a good hallmark for comparison of QOL across diseases, instruments developed specifically to measure vision-specific QOL may provide improved accuracy for assessing the impact of vision-threatening disease [11-14]. Future population-based studies using vision-specific QOL instruments are needed to investigate QOL in AMD patients.

When the current study began, we expected that a poor QOL (lower EQ-5D and EQ-VAS scores) would show a strong association with depressive symptoms in AMD patients. A simple comparison in AMD patients revealed significant differences for all EuroQol indices between people both with and without depressive symptoms. However, multivariate regression showed that only the "anxiety/depression" dimension of the EQ-5D was significantly associated with depressive symptoms. The indices representing comprehensive health-related QOL and the EQ-VAS and EQ-5D did not show a significant association with depressive symptoms. However, when the QOL as assessed by the "anxiety/depression" dimension was poor, the possibility of having depressive symptoms increased. This result could be because the question used to identify the depressive symptom group and the one used for the EQ-5D ("anxiety/depression") were similar. This aspect might also serve to explore a possible association between AMD and depression and to increase the statistical power of our study.

Depression and AMD must be handled comprehensively, not separately, as one can affect another. These conditions should be treated as a context within the larger framework of comorbid depression with chronic illness.

Comorbid depression can lead to increased morbidity of AMD as it compounds the disability resulting from vision loss and may even impact a patient's ability to seek out appropriate care. Several studies have shown that depression is linked to increased disease-related morbidity and mortality. This has been particularly well studied with chronic illness; depression has been shown to be clearly associated with a poorer prognosis and more rapid progression of chronic illnesses [27-29]. In ischemic heart disease and diabetes, physiologic changes may occur that are affected by depression and can increase morbidity in addition to decreased treatment adherence and mobility and increased substance abuse [27,28].

In addition, although these patients are at greater risk than the general population, having a chronic illness may actually make it less likely that providers will screen for depression given the demands of chronic illness management and coordination of care.

Furthermore, treating depression might change the outcomes of AMD. It was previously reported that a mental health intervention for patients with AMD halved the incidence of depression compared to those receiving standard treatment for AMD [30].

Our study had several limitations. First, AMD was diagnosed using fundus photography alone in the present study; no other ophthalmic examination method, including fluorescein angiography or optical coherence tomography, was carried out because of the nature of this large population-based survey. In the future, we will be able to assess ophthalmic risk factors in more detail and identify their specific effects on depressive symptoms by incorporating data from other ophthalmic examinations into our study. Second, a single question was provided to measure the degree of depressive symptoms instead of established depression questionnaire tools, so the accuracy of our responses could be limited. Further, this one question provided little 
information regarding the degree of severity of the depression or its relationship with the severity of AMD and level of dependency. However, the question "do you often feel sad or depressed?" showed a sensitivity of $86 \%$, a specificity of $78 \%$, and positive predictive and negative predictive values of $82 \%$ in previous studies, so the error is likely small [31]. Third, the screening for ADL was carried out with just a yes or no answer, and the question appears relatively non-specific. The question does not indicate the degree of dependency on ADL because there is likely variability in what individuals consider "disturbances" in everyday life. Fourth, since this was a cross-sectional study, a causal relationship could not be defined. Fifth, the possible influence of treatment with intravitreal anti-vascular endothelial growth factor injection for AMD and anti-depressants for depression was not considered. These factors could be used as covariates in a future multivariate analysis.

Despite these limitations, this study used representative nationwide, population-based data to determine the prevalence of depressive symptoms in Korean AMD patients. The current study was the first population-based study to evaluate depression and QOL in Korean AMD patients. Because the social, cultural, and racial/ethnic characteristics of Korean AMD patients may be different from those in Western countries, our evaluation of depression and QOL in Korean AMD patients is valuable. Our study indicated that female gender and dependency in ADL were the most important associated factors for depressive symptoms in Korean AMD patients. Ophthalmologists may consider using these findings to determine which patients should undergo further evaluation and treatment for depression, such as neuropsychiatric support.

\section{Conflict of Interest}

No potential conflict of interest relevant to this article was reported.

\section{Acknowledgements}

We would like to thank Lucia Sobrin, MD, MPH, for generous support of this study.

HYC had full access to the data in the study and takes responsibility for the integrity of the data and the accuracy of the data analysis.

\section{References}

1. Klein R, Klein BE, Jensen SC, Meuer SM. The five-year incidence and progression of age-related maculopathy: the Beaver Dam Eye Study. Ophthalmology 1997;104:7-21.

2. Pascolini D, Mariotti SP, Pokharel GP, et al. 2002 Global update of available data on visual impairment: a compilation of population-based prevalence studies. Ophthalmic Epidemiol 2004;11:67-115.

3. Kawasaki R, Yasuda M, Song SJ, et al. The prevalence of age-related macular degeneration in Asians: a systematic review and meta-analysis. Ophthalmology 2010;117:921-7.

4. Song SJ, Youm DJ, Chang Y, Yu HG. Age-related macular degeneration in a screened South Korean population: prevalence, risk factors, and subtypes. Ophthalmic Epidemiol 2009;16:304-10.

5. Casten R, Rovner B. Depression in age-related macular degeneration. $J$ Vis Impair Blind 2008;102:591-9.

6. Banerjee A, Kumar S, Kulhara P, Gupta A. Prevalence of depression and its effect on disability in patients with age-related macular degeneration. Indian J Ophthalmol 2008;56:469-74.

7. Brody BL, Gamst AC, Williams RA, et al. Depression, visual acuity, comorbidity, and disability associated with age-related macular degeneration. Ophthalmology 2001;108:1893-900.

8. Jivraj J, Jivraj I, Tennant M, Rudnisky C. Prevalence and impact of depressive symptoms in patients with age-related macular degeneration. Can J Ophthalmol 2013;48:269-73.

9. Rovner BW, Casten RJ, Tasman WS. Effect of depression on vision function in age-related macular degeneration. Arch Ophthalmol 2002;120:1041-4.

10. Lee WJ, Cho HY, Kim DH, et al. Depression of late age-related macular degeneration patients in Korea. Asia Pac J Ophthalmol (Phila) 2013;2:23-7.

11. Cahill MT, Banks AD, Stinnett SS, Toth CA. Vision-related quality of life in patients with bilateral severe age-related macular degeneration. Ophthalmology 2005;112:152-8.

12. Coleman AL, Yu F, Ensrud KE, et al. Impact of age-related macular degeneration on vision-specific quality of life: follow-up from the 10-year and 15-year visits of the Study of Osteoporotic Fractures. Am J Ophthalmol 2010;150:683-91.

13. Piermarocchi S, Varano M, Parravano M, et al. Quality of vision index: a new method to appraise visual function changes in age-related macular degeneration. Eur J Oph- 
thalmol 2011;21:55-66.

14. Slakter JS, Stur M. Quality of life in patients with age-related macular degeneration: impact of the condition and benefits of treatment. Surv Ophthalmol 2005;50:263-73.

15. Korea Centers for Disease Control and Prevention. Korea National Health and Nutrition Examination Survey raw material guideline [Internet]. Cheongju: Korea Centers for Disease Control and Prevention; 2010 [cited 2017 Jun 20]. Available from: http://knhanes.cdc.go.kr/.

16. Ha JY, Kim ES, Kim HJ, Park SJ. Factors associated with depressive symptoms in patients with chronic low back pain. Ann Rehabil Med 2011;35:710-8.

17. Kim SH, Kim JS, Choi JY, et al. Factors associated with depressive symptom in chronic obstructive pulmonary disease based on the third (2005) and fourth (2008) Korea National Health and Nutritional Examination Survey (KNHANES III, IV). Korean J Fam Med 2011;32:234-42.

18. Brooks R. EuroQol: the current state of play. Health Policy 1996;37:53-72.

19. EuroQol Group. EuroQol: a new facility for the measurement of health-related quality of life. Health Policy 1990;16:199-208.

20. Nam HS, Kim KY, Kwon SS, et al., editors. EQ-5D Korean valuation study using time trade off method. Seoul: Korea Centers for Disease Control and Prevention; 2007.

21. Bird AC, Bressler NM, Bressler SB, et al. An international classification and grading system for age-related maculopathy and age-related macular degeneration. The International ARM Epidemiological Study Group. Surv Ophthalmol 1995;39:367-74.
22. Jin YH. A new logMAR vision chart: Jin's vision chart. $J$ Korean Ophthalmol Soc 1997;38:2036-44.

23. Blazer DG. Depression in late life: review and commentary. J Gerontol A Biol Sci Med Sci 2003;58:249-65.

24. Cho MJ, Lee JY, Kim BS, et al. Prevalence of the major mental disorders among the Korean elderly. J Korean Med Sci 2011;26:1-10.

25. Kim JM, Stewart R, Shin IS, et al. Lifetime urban/rural residence, social support and late-life depression in Korea. Int J Geriatr Psychiatry 2004;19:843-51.

26. Cho MJ, Nam JJ, Suh GH. Prevalence of symptoms of depression in a nationwide sample of Korean adults. Psychiatry Res 1998;81:341-52.

27. de Groot M, Anderson R, Freedland KE, et al. Association of depression and diabetes complications: a meta-analysis. Psychosom Med 2001;63:619-30.

28. Glassman AH, Shapiro PA. Depression and the course of coronary artery disease. Am J Psychiatry 1998;155:4-11.

29. Simon GE. Treating depression in patients with chronic disease: recognition and treatment are crucial--depression worsens the course of a chronic illness. West $J$ Med 2001;175:292-3.

30. Rovner BW, Casten RJ, Hegel MT, et al. Low vision depression prevention trial in age-related macular degeneration: a randomized clinical trial. Ophthalmology 2014;121:2204-11.

31. Watkins C, Daniels L, Jack C, et al. Accuracy of a single question in screening for depression in a cohort of patients after stroke: comparative study. BMJ 2001;323:1159. 